\title{
A Different Approach in an AAL Ecosystem: a Mobile Assistant for the Caregiver.
}

\author{
Angelo Costa ${ }^{1, *}$, Oscar Gama ${ }^{2}$, Paulo Novais ${ }^{1}$, Ricardo Simoes ${ }^{2,3,4}$ \\ ${ }^{1}$ CCTC-Computer Science and Technology Center, University of Minho, Braga, Portugal \\ \{acosta, pjon\}@di.uminho.pt \\ ${ }^{2}$ Institute for Polymers and Composites - IPC/I3N, University of Minho, Campus de Azurém, \\ 4800-058 Guimarães, Portugal \\ osg@i.uminho.pt, rsimoes@dep.uminho.pt \\ ${ }^{3}$ Life and Health Sciences Research Institute (ICVS), School of Health Sciences, University of \\ Minho, Campus de Gualtar, 4710-057 Braga, Portugal \\ ${ }^{4}$ Polytechnic Institute of Cavado and Ave, Campus do IPCA, 4750-810 Barcelos, Portugal \\ rsimoes@ipca.pt
}

\begin{abstract}
Currently the Ambient Assisted Living and the Ambient Intelligence areas are very prolific. There is a demand of security and comfort that should be ensured at people's homes. The AAL4ALL (ambient assisted living for all) project aims to develop a unified ecosystem and a certification process, allowing the development of fully compatible devices and services. The UserAccess emerges from the AAL4ALL project, being a demonstration of its validity. The UserAccess architecture, implementation, interfaces and test scenario are presented, along with the sensor platform specially developed for the AAL4ALL project.
\end{abstract}

Keywords: Ambient Assisted Living; Ambient Intelligence; Intelligent Environments; AAL4ALL, e-Health, Active Ageing; Artificial Intelligence; Sensor Platform

\section{Introduction}

In the last 20 years modern society has witnessed several technological evolutions, as the birth of high speed internet, smartphones, GPS systems, smart TV's, among others devices [1]. Furthermore, we can state that the proliferation of these devices on a home environment induced behavioural changes in the youngest generations. Even older people have progressively accepted and adopted these devices on their work or at home, thus, we can say that society considers these devices as a common good and being always present [2]. Moreover, with the current advances, some devices have transcended from being tools to perform a task to being the motive of the human interaction with them. As an example, the smartphone has started as work facilitator tool, providing everywhere internet access and office tools, to become an entertainment centre, with music, videos and games. Even the way people communicate has changed due to these devices, shaping the language and the social interaction between humans $[3,4]$. 
Historically, the main driving forces behind the evolution of these technologies were the medical, military and engineering fields. To solve complex problems they require pioneering technology, which is obtained through massive investments [5]. Even spinoff products are of use and important, if not to the area they were produced, to other areas, branching into other lines of investigation. Also, the people who adopted emerging technology contributed greatly to the investment in home version of industrial devices.

Home automation is one field that displayed abundant progression due to the adoption of technology developed to other fields. The crescent use of automated devices on common homes have forced a cost reduction of them, thus allowing a greater panoply of choices in terms of features and complexity. The merge of home automation devices with computational systems resulted in the Ambient Intelligence concept (AmI).

AmI aim is integrating all devices in an ecosystem and the obtaining the environment's context $[6,7]$. These two goals can be described in the following way:

- Integration: allows different devices, such as sensors and actuators, to communicate with the rest of them, being communication protocol agnostic. The homogenization of the communication protocol leads to the possibility of using devices from different makers, or easily changing the device's topology and placement. The ecosystem created is permeable to changes and allows new additions to the network;

- Context: enables the capture of information relative to the environment's state. Simple sensor information only provide a limited spectrum of the environment. Context is usually the outcome of information fusion, which provides a broader perspective of the environment. Therefore, complex information can be obtained from simple sensor data, in some cases outlining unexpected states that each sensor by itself was unable to do. Context also places itself on high-level communicating process, transmitting clear information that encapsulates simple actions.

The evolution of the AmI was allowed due to the four main aspects:

- Device miniaturization: allowing complex systems be encapsulated in a small form factor;

- Information availability: datasets from heterogeneous sources and classification of said data allowed the generation of knowledge that can be implemented directly on the developing systems.

- Computing power: allowing the ability to consume information and processing it faster than ever before, obtaining results instantaneously;

- Ubiquity: allowing integration of computerized system in common appliances, providing them with the capability of communication and integration with the ecosystem, transforming them in a sensor/actuator terminal.

AmI's are structured to be constructed over very different scenarios, such as home environments, offices, industrial factories, hospitals, day-care centres, among others. Although this architectural feature is important, allowing everyone access to this technology, it presents an implementation problem. It is easy to grasp that a hospital environment is very different of a factory environment, and so are the requirements of each of 
them. Thus, the adaptation to each environment that is going to be implemented is essential, and the areas that care for the home and medical environments is named Ambient Assisted Living (AAL).

\subsection{AAL}

The AAL concept is fundamentally the one of the AmI, but with the directed towards a specific population and environments, being mainly the elderly and the people with disabilities [8-12]. They require special attention due to their limitations leading the systems to be sensible enough to accommodate them properly. Adaptation is key in these cases and the AAL provide it. As the name shows, the AAL can be defined by a sentient environment that helps sensibly the people in it, on their daily living $[13,14]$.

To be sentient, a system has to be intelligent. Alike humans, which are able to perceive a scenario and sort the elements and events happening, these systems must also have to be able to capture and contextualize the information obtained by the sensors.

Sensor systems are very useful as they provide an array of sensors, able to capture different environment aspects, but they also present a flaw, they are only able to capture the information according to their specification, and unable to distinguish the "bigger picture". Here, the sum of all parts are usually greater than the parts themselves, where the perspective is fundamental, so having only streams of data it is very difficult, if not impossible, to describe what is really happening in the environment. For instance, if a flood sensor is not paired with a fire alarm, in an event of a small fire that could be easily contained, a flood could occur, damaging valuable propriety.

What currently happens is that most of the home systems rely only on simple logic systems, implemented directly on the system middleware. This approach a proven solution directed for simple smart homes' implementations, with only a few features. A problem emerges when the users and the environments are more exigent. Thus, a simple system is just not enough to respond to the demands.

The AAL aims to provide personalised systems that adapt to the user, not only on implementation but along the user's life. Furthermore, the intended users of the AAL have very specific and complex disabilities so they must have environments that are designed to them, helping them on their daily tasks.

In this paper we present the AAL4ALL project and an AAL system that was developed to validate the project concept.

\section{UserAccess: An AAL4ALL project}

The AAL4ALL project [15] is a Portuguese effort on the AAL area, being consortium of private companies and public institutions. The aim is to deliver solutions, in the form of devices or services, and a certification process so that other developers (outside the consortium) can obtain a national approved for sale seal. Being an AAL project, the AAL4ALL goal is to ease the life of the elderly and disabled by offering an ecosystem adjusted to them, having into account the social and monetary conditions. 
The distinctive feature from other projects is the ecosystem's integration. The integration procedures were assured since the project conception, thus, a large effort was done by all project partners to construct products and services that were compliant with the rest. Additionally, the project relied on a social sphere created to support the user constituted by formal and informal caregivers, and family and friends. It is clear that some procedures must be accompanied by humans, justifying the need to resort to caregivers, so allowing the inclusion of caregiving companies.

The core architecture of the project is open, allowing access to anyone that desires developing a solution to it. The components heterogeneity enforces the integration of them, and the homogeneity of the final product requires that every device must be able to trade information as effectively and as perceivable as possible.

One of the AAL4ALL solutions is the UserAccess, consisting in a devices and service structure, aimed mainly to the caregivers, creating a digital bridge between the user and the caregiver.

\subsection{UserAccess}

The UserAccess project is an AAL implementation based on but presenting a different approach of the project shown in $[14,16]$. The project's goal is to deliver user information to the caregiver, thus assisting the caregiver on him/her daily task, by retrieving information from sensor platforms and formulating knowledge about the environment's context.

Most of the AAL projects rely on a human assistance network, which provide help on critical situations or by user demand. They act as a medical proxy, providing assistance and social support remotely so the user can stay at home and trial the simpler cases without needing to go to a hospital constantly. The main issue with these services is that they require on the user interaction, meaning that the usually is the user who must phone them if there is any case wrong. They act very passively and in the critical cases are little to no user of the user. In the last years there was a proliferation of "panic buttons" consisting of a hardware device that had a unique button and with a press it calls the caregiving services or the emergency medical services. Additionally to passing the user information to the services, the panic button could establish a phone call or text messaging, resulting on a few different services that can be offered, depending on the device capabilities. The point is that none of them (caretaking services and panic buttons) are truly a good solution to the user. One is unable to act in critical situations, and other acts only when a critical situation is ongoing.

In this project the importance of the caretaking services is acknowledged, as we consider that the human supervision it is still the best approach when medical related events happen. With this in mind, the UserAccess is built to help caregivers, and may be modestly explained as: receiving the information from the house's sensors system, process it and deliver it to the caregiver in human-readable form. The system is constituted by a server and a mobile platform, preferably a smartphone or tablet and can monitor several users at the same time. 
The information that is presented to the caregiver is related to the context rather than to each sensor, although some sensors (e.g. sos button) are directly reported in form of a notification, with the maximum priority possible.

Architecture. As stated before, the UserAccess is constituted by a software service and hardware devices, thus the architecture of the project also reflects those features. The UserAccess follows the integration standards that were defined on the AAL4ALL, and because it is an AAL4ALL project it uses its structure to communicate.

The communication is assured be the AAL4ALL Node which serves as a communication bridge, providing channels form publishing and consuming information. Due to the heterogeneity of the sensor systems the sustaining platform requires that they communicate at higher level information, thus, the sensor platform has to provide a middleware component to process the raw data.

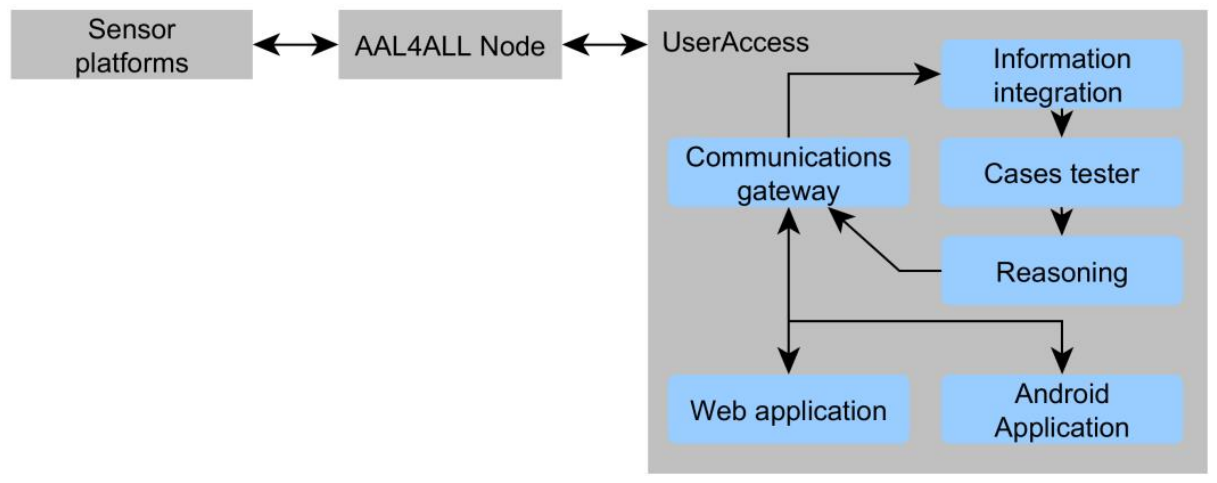

Fig. 1. UserAccess architectural components.

As depicted in the Figure 1, the UserAccess receives high-level information, such as "user exited home" or "lights are on" trough the Communications gateway, which performs a verification of the incoming data checking the validity and restructures it according the internal communication method. Thereon, the information sent to the Information integration and is merged with the previous user state, updating the environment's context, forming new knowledge about the user's actions.

The context aspect is essential, thus a vector is formed containing the previous knowledge, the new knowledge and the base rules. In the Cases tester the vector is tested to verify if it is already in the knowledge base, and if any response was previously defined. If a case is present (according to the similarity) then same approach is used, but if it is not present the event is saved for further analysis.

The next step is the Reasoning, and it contains the previous actions and a similarity analysis process that compares the cases and retrieves them along with the rules, obtaining the action to be performed. Finally, the set of actions are transformed into information or notifications to send to the caregiver mobile platform.

The Cases tester and Reasoning are based over Case Based Reasoning and Bayesian Networks concepts, as in this project were considered as the best approach due to the 
information type and the relational knowledge. Also, what is called action on the UserAccess process is a bundle of events that must be performed by the interfaces, such as displaying certain messages or notifications, as shown in Figure 2. A bilateral communication process was implemented to validate the test scenario consisting in an electrical switch actuator that was responsible of turning on or off a light.

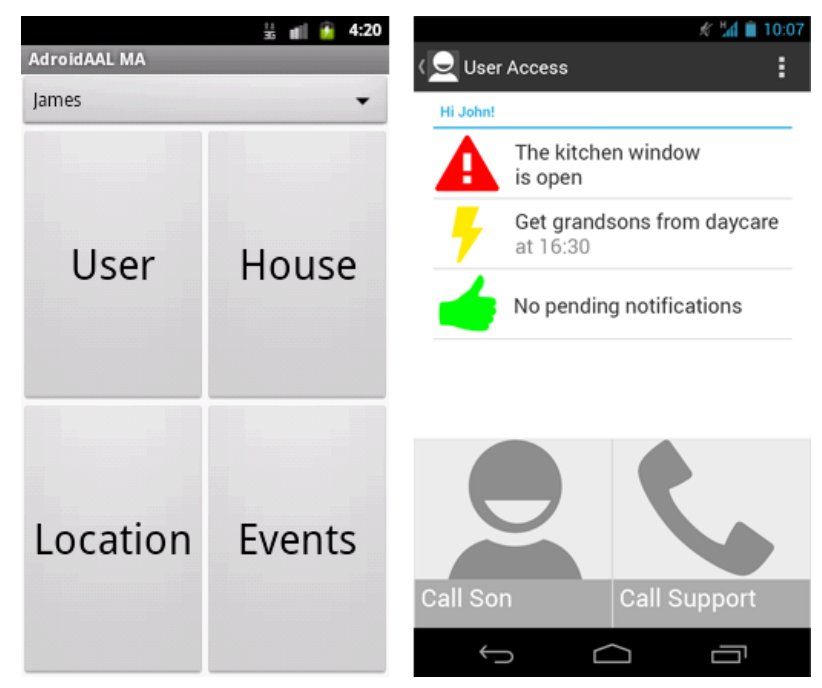

Fig. 2. a) The Android application home interface. b) Alpha stage caregiver interface (warnings and activities).

Scenario. At the current stage of the development the system must undergo on tests performed in a controlled environment, using the sensor platform and a local server. It is critical that a finished product is presented to the user, thus it is critical having a testing process recurring to predefined scenarios.

The sensors used are shown in the Figure 3. They use the ZigBee protocol to communicate, and possess a base station connected to a Raspberry Pi computer, acting as middleware. The sensors displayed on the Figure 3 are: (on the front) two motion sensors, a light and environment temperature and relative humidity sensor, two door/window open/close sensor, a SOS button, and on the back the electrical switch actuator.

The base station received the information from these devices and communicated with the middleware, being the speed of the communication negligible as it was immediate. The middleware also has enough power to process raw data and transform it into information accepted by the server. The middleware saves the direct previous states $(5$ to 10) to deliver stable information and to filter errors. Furthermore, it also detects the sensor's battery level and connection quality.

The server is constructed following a multi-agent system concept, being constituted by several modules that are responsible for the knowledge process. Currently the system is unable to form new knowledge, being the information saved in a knowledge base and after each test the WEKA tool is used to find correlations. 
Five tests were performed in the proposed scenario, each with 10 attempts, being the following list:

- Test 1: the user gets up from a seated position and exits the door;

- Test 2: the user gets up from a seated position and open and closes the door;

- Test 3: the user enters the house;

- Test 4: the user gets close to the door but does not open it;

- Test 5: Press the SOS button.

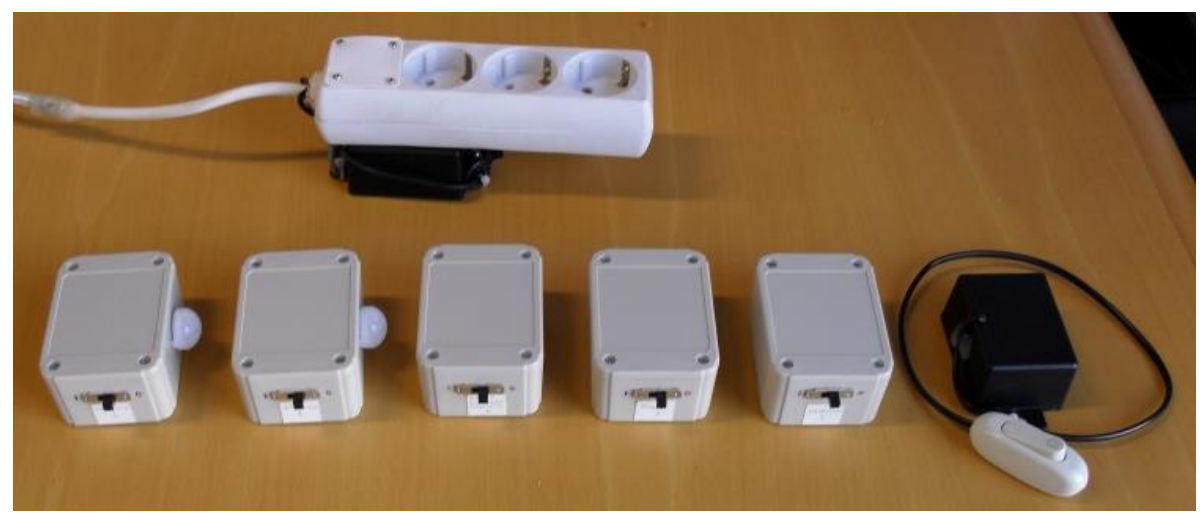

Fig. 3. Sensors used in the scenario setup.

Of those tests, the system was able to correctly detect the procedures (7 of 10) having difficulty when noise was introduced in some of the events. In all cases the caregiver received a notification and a threat assessment. In the failed tests, the main issues were caused by the sensor's latency, causing a deviation to the expected event. To perform these tests a logic construction was devised and implemented in each agent. For instance, to detect the user going outside the agent monitored the sensors that retrieved that information, usually being more than one, and in this particular case, three (the two motion sensors and the door sensor).

As stated before, the stabilization time is an issue that directly impacts the states detection and even the actions to be performed. For instance, the motion detection sensors take 5 to 10 seconds to stabilize, which means that for that period of time the system has no idea of what is happening. For instance, if the user has full mobility he/she could open the door, exited, go back inside and stand still until the sensors stabilize and in the best case scenario the system would detect that the user is still inside. This perilous example shows the dependency of the software has over the information of the hardware. In our perspective, a compromise can be reached to overcome the stabilization problem, having two solutions: a redundant system, or other sensor formats.

Other sensor formats means that, for instance, for the motion detection, the current sensors should be replaced by other sensors, such as floor sensors, cameras or even laser sensors. The major issue with this solution is that we aim to have a sensor platform that is monetarily accessible thus, the use of high-end sensors rapidly inflates the expected budget and compromises the acceptance by the users. 
The redundant set of sensors consists in equipping the environment with more sensors, being the same type or other cheap ones. This way in addition to have motion detection inside a house, a few more can be place outside the house or even placed in other angles. This solution is the most inexpensive and, with a fine tuned logic associative procedure, can in fact outperform the system with more advanced but with less number of sensors. With different stabilization times is expected that the software will be receiving information at all times thus covering the shortcomings of each sensor. In the case of the motion detection sensors, in the Figure 4 is showed the current setup (in the left) and the right side the possible setup of an entry hall.
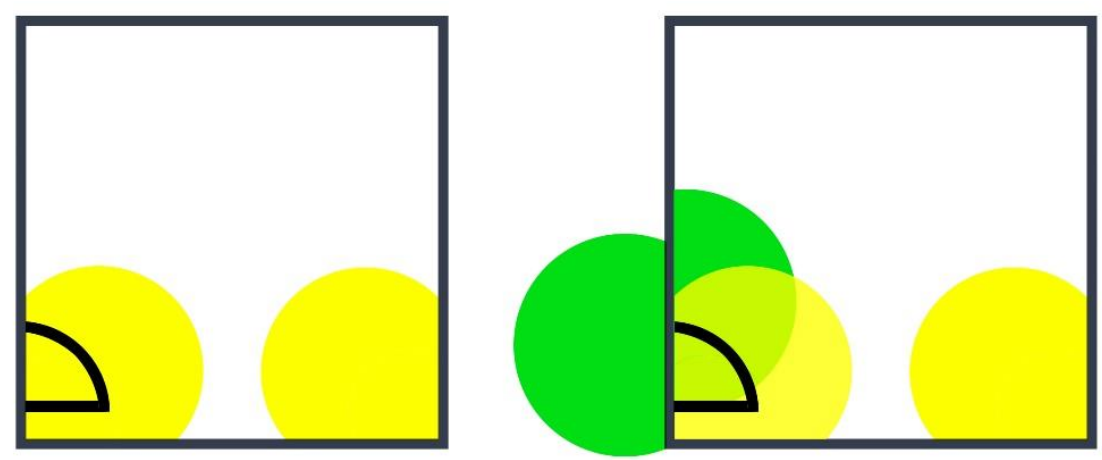

Fig. 4. a) Current motion sensor positioning. b) Advanced motion sensor positioning.

In Figure 4, on the b) image, it is clear that the additional information about the motion can easily provide full information about the user location. As the motion detection sensors only act when the user is moving they do not know where they are within the scope of the sensor. Thus with several sensors it is possible to verify the several activation sequence and stabilization procedure thus, we can obtain the exact user action.

A similar conclusion can be obtained to all other sensors, for instance, in terms of temperature, light and electrical switches. They are inexpensive and easy to implement, thus the number of available devices can be greatly increased, increasing also the quality of the obtained information.

The UserAccess agent-based architecture is able to easily support sensor additions to the platform, being the requirement only to comply with the API and the communication ontologies. The agents can be updated to find other sensors of the same type and the logical procedures adapted to receive blocks of information with the only requirement of setup the sensor sequences to each action.

\section{Conclusions and Future Work}

In this paper was presented the AAL4ALL project and the UserAccess project. The AAL4ALL project is already an important landmark, presenting novelty features such 
as the integration methods and the certification procedures. The UserAccess is an effort towards the validation of the AAL4ALL concept, among the other partners' projects.

The AAL4ALL project is now shifting towards an integrated solution, being the aim to implement all the sensor communications and early processing to a set-top box, which presents the information locally in a television. The heavy data processing will be done on the cloud, where the UserAccess is expected to be placed. The aim of the UserAccess remains the same, being to display the information to the user and the caregiver, in a functional webpage. Moreover, the agent-base system is also maintained, being currently adapted to receive high-level information, as the initial processing system is placed on the home set-top box. This releases the UserAccess of the sensor data processing tasks to being more focused in providing real actions relations, being able to provide personal information and a profile. We are aiming to detect potentially hazardous actions and provide information to the caregiver about them, allowing to act preventively.

In terms of future architecture and technical work, we expect to implement a load balancing feature on the server, and an optimized interface structure. Establishing a better communication protocol between the server and the mobile platform, optimizing the message weight and establishing ontologies to structure all content.

In terms of the sensor platform, the current challenges are the miniaturization and the battery life. To proceed to a field test, we need to use devices that are nearly at the end-product phase so the invisibility and duration are key factors to a successful experiment, allowing a smooth and functional operation.

Currently, we can state that the UserAccess is at an advanced stage, being the architecture and most of the modules developed. The reasoning of the system is undergoing on several developments and is expected to be operational in the near future.

\section{Acknowledgements}

Project "AAL4ALL", co-financed by the European Community Fund FEDER, through COMPETE - Programa Operacional Factores de Competitividade (POFC). Foundation for Science and Technology (FCT), Lisbon, Portugal, through Project PEstC/CTM/LA0025/2013 and the project PEst-OE/EEI/UI0752/2014.

Project CAMCoF - Context-aware Multimodal Communication Framework funded by ERDF -European Regional Development Fund through the COMPETE Programme (operational programme for competitiveness) and by National Funds through the FCT - Fundação para a Ciência e a Tecnologia (Portuguese Foundation for Science and Technology) within project FCOMP-01-0124-FEDER-028980.

\section{References}

1. Cozza, R., Milanesi, C., Zimmermann, A., Glenn, D., Gupta, A., Shen, H.J.D.L.V., Lu, C., Sato, A., Huy, T., Sandy, N.: Market Share: Mobile Communication Devices by Region and Country, 3Q11. (2011).

2. Markopoulos, P., Mavrommati, I., Kameas, A.: End-User Configuration of Ambient Intelligence Environments: Feasibility from a User Perspective. Ambient Intelligence. 243-254 (2004). 
3. Shuler, C.: Industry Brief: Pockets of Potential: Using Mobile Technologies to Promote Children's Learning. (2009).

4. Common Sense Media: Zero to Eight: Children's Media Use in America 2013. (2013).

5. Jorge, J.A.: Adaptive tools for the elderly. Proceedings of the 2001 EC/NSF workshop on Universal accessibility of ubiquitous computing providing for the elderly WUAUC'01. p. 66. ACM Press, New York, New York, USA (2001).

6. Russell, S., Norvig, P.: Artificial Intelligence: A Modern Approach. Prentice Hall (1995).

7. $\quad$ Augusto, J.C., Callaghan, V., Cook, D., Kameas, A., Satoh, I.: Intelligent Environments: a manifesto. Human-centric Computing and Information Sciences. 3, 12 (2013).

8. Kurschl, W., Mitsch, S., Schönböck, J.: Modeling Situation-Aware Ambient Assisted Living Systems for Eldercare. 2009 Sixth International Conference on Information Technology: New Generations. pp. 1214-1219. IEEE (2009).

9. O’Grady, M.J., Muldoon, C., Dragone, M., Tynan, R., O’Hare, G.M.P.: Towards evolutionary ambient assisted living systems. Journal of Ambient Intelligence and Humanized Computing. 1, 15-29 (2009).

10. Botia, J.A., Villa, A., Palma, J.: Ambient Assisted Living system for in-home monitoring of healthy independent elders. Expert Systems with Applications. 39, 8136-8148 (2012).

11. Nehmer, J., Becker, M., Karshmer, A., Lamm, R.: Living assistance systems: an ambient intelligence approach. Proceedings of the 28th international conference on Software engineering. 43-50 (2006).

12. Novais, P., Costa, R., Carneiro, D., Neves, J.: Inter-organization cooperation for ambient assisted living. Journal of Ambient Intelligence and Smart Environments. 2, 179-195 (2010).

13. Lima, L., Novais, P., Costa, R., Bulas Cruz, J., Neves, J.: Group decision making and Quality-of-Information in e-Health systems. Logic Journal of IGPL. 19, 315-332 (2010).

14. Costa, Â., Castillo, J.C., Novais, P., Fernández-Caballero, A., Simoes, R.: Sensordriven agenda for intelligent home care of the elderly. Expert Systems with Applications. 39, 12192-12204 (2012).

15. AAL4ALL - Ambient Assisted Living For All, www.aal4all.org.

16. Vardasca, R., Costa, A., Mendes, P.M., Novais, P., Simoes, R.: Information and Technology Implementation Issues in AAL Solutions. International Journal of EHealth and Medical Communications. 4, 1-17 (2013). 